\title{
Oral changes in Diabetes - a review
}

\author{
Ngairangbam Sanjeeta ${ }^{1}$ \\ ${ }^{I}(M D S$, Assistant Professor, Dental College, Regional Institute of Medical Sciences, Imphal))
}

\begin{abstract}
Diabetes mellitus is a chronic disease affecting all age groups and one of the leading causes of mortality and morbidity worldwide. Many chronic macrovascular and microvascular complications of diabetes have been reported in the literature with few reports on diabetes and associated various oral soft tissue abnormalities. In this review, the known correlations between oral disease and diabetes mellitus are highlighted with the aims to increase the awareness of oral changes, complications of diabetes mellitus and to emphasize on need of studies to control these complications. It treats in depth some of the complications such as periodontal disease, fungal infection and salivary dysfunction.
\end{abstract}

Keywords: Diabetes Mellitus, Oral, Complications, Taste, Fungal.

\section{INTRODUCTION}

The countries with the largest number of diabetes are India, China and the US, with India beginning to be regarded as the diabetic capital of the world. ${ }^{1}$ The chronic hyperglycemia and attendant metabolic dysregulation in diabetes mellitus (DM) may be associated with secondary damage in multiple organ systems, especially the kidneys, eyes, nerves and blood vessels. ${ }^{2}$ The association between DM and changes in the oral cavity have been the subject of reports in both the medical and dental literatures. A number of oral soft tissue abnormalities have been associated with DM by various reports, with more emphasis being given on its relation with periodontal diseases. ${ }^{3}$ There are strong evidence of both type 1 and type 2 diabetic patients having more severe periodontal disease than do individuals without diabetes. ${ }^{4-7}$ Other specific conditions that have been identified include geographic tongue, fissured tongue, median rhomboid glossitis, hyperplastic gingivitis, lichen planus, parotid gland enlargement, candidiasis, xerostomia, burning sensations of oral mucosa, taste disturbances, increased incidence and severity of dental caries, traumatic ulcers, etc. ' 'Grinspan syndrome' is an interesting condition in which there is coexistence of DM, lichen planus and hypertension in the same individual.

\section{Pathogenesis}

The underlying cause for increased susceptibility to infection, impaired wound healing capabilities and vascular injury following chronic hyperglycemia in DM can be attributed to various underlying mechanisms. ${ }^{2}$ The underlying mechanisms ${ }^{2,8}$ which, in turn, affect infections can be associated with

- Sorbitol- myoinositol osmolarity changes

- Oxidative- redox stress

- Non-enzymatic glycation reactions (advanced glycation end products- AGEs)

- Activation of the protein kinase C- diacylglycerol pathway.

The diabetic state impairs the synthesis of collagen and glycosaminoglycans in the gingiva, enhances crevicular fluid collagenolytic activity and results in the loss of periodontal fibres, supporting alveolar bone, loosening and exfoliation of the teeth. ${ }^{9}$

Diabetes- induced changes in immune cell function produces an inflammatory immune-cell phenotype (upregulation of proinflammatory cytokines from monocytes/ polymorph nuclear leukocytes and downregulation of growth factors from macrophages), predisposing to chronic inflammation, rogressive tissue breakdown, and diminished tissue repair capacity. ${ }^{9}$

\section{Sialosis}

Sialosis which is defined as an asymptomatic, non-inflammatory, non-neoplastic enlargement of the salivary glands due to metabolic causes has been observed frequently in diabetic patients, with one report involving $24 \%$ of the diabetic subjects. Sialosis is caused by fatty infiltration of the interstitium and enlargement of the acinar cells and, in diabetics, is characterized by bilateral enlargement of the parotid salivary glands though the submandibular gland may also be involved. Compensatory hyperplasia of the glandular parenchyma following a declining plasma insulin concentration, and a compensatory mechanism to combat xerostomia in diabetics has been interpreted by investigators as the causes of the glandular enlargement. ${ }^{10}$ 


\section{Xerostomia}

Xerostomia is a subjective sensation of the dryness of the mouth and has been a feature of uncontrolled diabetes. Xerostomia in diabetes is traced to decreased salivary secretion related to various factors associated with diabetes. Microvascular disease, autonomic neuropathy, dehydration and loss of urinary electrolytes resultant to polyuria are considered as causative mechanisms for the decreased salivary secretion in diabetics. ${ }^{10}$ Experimental studies in diabetic rats have shown that fatty infiltration and degeneration of the parotid gland destroy the secretory tissue leading to diminished saliva production. ${ }^{10}$ However, more than a third of diabetic patients report xerostomia in the presence of normal production of saliva with some studies reporting comparable or increase in volumes of salivary secretion. Hence, the pathogenesis of this condition still remains unclear and may have a psychological component. ${ }^{10}$

\section{Taste impairment}

All the primary taste sensations may be blunted as is demonstrated by chemical gustometry. Taste threshold may also be altered and is demonstrable by electrogustometry. Impairment of the sweet taste has been most frequently reported and may be present at diagnosis. Although taste impairment is usually well tolerated, a blunted sweet sensation may favour sweet tasting food and may exacerbate the hyperglycemia in an undiagnosed diabetic patient. The role of altered taste sensation in the predilection for food containing refined sugar and the subsequent poor dietary compliance in some diabetics has not been investigated. Sulphonylureas, the group of drugs commonly used type 2 diabetes have been shown to be associated with altered taste sensation $^{10}$.

\section{Oral lichen planus and lichenoid reaction}

Lichen planus is a mucocutaneous disorder of uncertain aetiology. An association between oral lichen planus (OLP) and impaired glucose tolerance has been reported, but may be coincidental as it has not been confirmed by various investigators. OLP may either regress spontaneously or may respond to local treatment unrelated to the glycemic state in DM. The triad of OLP, DM and hypertension in Grinspan's syndrome may be a coincidental association. What is clinically diagnosed as lichen planus could perhaps be a lichenoid reaction developing as a side-effect of certain oral hypoglycemic or antihypertensive drugs ${ }^{10-11}$.

\section{Oral candidosis}

Candida species have frequently been reported to be more prevalent and have been isolated in greater numbers from the oral cavity of diabetics compared to non-diabetic individuals. Enhanced growth of the yeast may be attributed to low salivary secretion and presence of a high concentration of salivary glucose. Subjects with diabetes were found to be at least five times more likely than control subjects to have oral soft tissue disease attributable to candidiasis. Prerequisites for candidal infections like enhanced adhesiveness of candida to oral epithelium and an impaired immune response due to defective neutrophil function is a feature of diabetes ${ }^{10}$.

\section{Dental caries}

Saliva and gingival crevicular fluid, in addition to constituents of the diet are the source of fermentable carbohydrates required for the growth of cariogenic bacteria. Significantly elevated levels of glucose in saliva and crevicular fluid, along with decreased salivation as they occur in diabetics may promote the increase in the incidence of dental caries. Although the total oral intake of refined carbohydrates may be reduced in diabetic patients, ingestion of food still occurs at frequent intervals throughout the day, and causes a repeated reduction in the $\mathrm{pH}$ of the oral cavity which is another factor for the increased susceptibility ${ }^{10}$.

\section{Mucormycosis}

It is a rare but serious systemic fungal infection that may occur in uncontrolled diabetes. Mucormycosis is an aggressive, frequently fatal invasive fungal infection that can develop in immunocompromised patients. Mucor is often recognized as a triad of symptoms, such as uncontrolled diabetes mellitus, periorbital infection and meningoenchephalitis ${ }^{13}$.

\section{Tongue changes}

Various studies report increased incidence of median rhomboid glossitis, benign migratory glossitis, burning mouth syndrome or fissuring of the dorsum of the tongue.

Median rhomboid glossitis which is a painless, central, depapillated area on the middle third of the dorsum of the tongue has been reported in $30 \%$ of 175 diabetic patients surveyed. ${ }^{10}$

There has been a four fold increase in incidence of geographic tongue compared to non diabetes according to a survey $^{10}$. In non-diabetic patients this lesion responds to systemic zinc therapy. 
Burning mouth syndrome which particularly affects the tongue and reported in subjects with undiagnosed diabetes are not related primarily to candidal infection and almost always resolves when glycemic control is instituted. Acute neuritis, chronic neuropathic lesion and xerostomia seen in diabetics are implicated in the aetiology, highlighting its multifactorial origin.

Fissured tongue was present in $28.0 \%$ in a survey of 175 diabetics ${ }^{12}$. Majority of them showed varying degree of placation with a transverse component to the fissures. Symmetrical, medially placed, double, longitudinal fissures extending from sulcus terminalis, meeting centrally to demarcate a triangular area of tongue were seen in some instances.

\section{Traumatic ulcers and irritation fibromas}

Higher prevalence for oral ulcers and irritation fibromas are reported in diabetics. Several mechanisms like slower healing time, microangiopathy or a defective function of the polymorph nuclear leukocytes may be responsible ${ }^{3}$.

\section{Periodontium and DM}

The relationship between periodontal disease and DM has been extensively studied and has been definitive enough to consider periodontitis as the sixth complication of DM. ${ }^{14}$ In diabetes with poor glycemic control, gingival and periodontal inflammations are manifested as increase in incidence and severity, with deep periodontal pockets, rapid bone loss, and frequent periodontal abscess. There has also been a correlation between the severity of the periodontal destruction, the duration of the diabetes and the presence of diabetic complications. Defective polymorphonuclear leukocyte function with resultant susceptibility of infection, increased collagenase activity, decreased synthesis and maturation of collagen and extra cellular matrix are postulated as causes of poor periodontal health in diabetics. The abnormal state of the collagen is attributed to excessive formation of advanced glycation end products (AGEs) in hyperglycemic states, which crosslink the collagen rendering it less soluble and hence less likely to be repaired or replaced. ${ }^{8}$

Tendency towards enlarged gingiva, sessile or pedunculated gingival polyps, polypoid gingival proliferations, abscess formation, periodontitis, and loosened teeth are the other varieties of periodontal changes described in DM.

\section{Conclusion}

The oral changes in diabetes are less likely to be seen in well controlled diabetics. The changes are not specific or pathognomonic for diabetes. The association of specific oral diseases and diabetes is not only of importance in the detection of undiagnosed diabetes, but also in the elucidation of the pathogenesis of various oro-facial diseases. However, the association of certain oral conditions with DM is not definitive and is still a matter of debate. In view of the ever increasing sedentary lifestyles and poor eating habits that have contributed to the escalation of DM worldwide, DM-related oral diseases deserve adequate recognition and further investigation.

\section{References}

[1]. Sharma A, Tiwari A. Diabetes mellitus and Dental Disease-a review. JIDA 2002; 73: 116-21.

[2]. Alvin C Powers. Diabetes Mellitus. In: Fauci AS, Braunwald E, Kasper DL, Hauser SL, Longo DL, Jameson JL, Loscalzo J, editors. Harrison's Principles of Internal Medicine. $17^{\text {th }}$ Ed. United States of America:McGraw-Hill;2008: 2275 - 304.

[3]. Guggenheimer J, Moore PA, Rossie K, Myers D, Mongelluzzo MB, Block HM. Insulin-dependent diabetes mellitus and oral soft tissue pathologies. I. Prevalence and characteristics of non-candidal lesions. Oral Surg Oral Med Oral Pathol Oral Radiol Endod 2000; 89 (5): 563 -69.

[4]. Finestone AJ, Bouriji. Diabetes mellitus in periodontal disease. Diabetes 1967:10:336 - 40.

[5]. Cerda J. Periodontal disease in NIDDM. The effect of age and time since diagnosis. J Periodontol 1995; 65: $991-95$.

[6]. Campus G, Salem A, Uzzau S, Baldoni E, Tonolo G. Diabetes and periodontal disease: A case control study. J Periodontol 2005; 76 (3): $418-25$.

[7]. Shlossman M, Knowler WC, Pettit DJ, Genco RJ. Type 2 DM and periodontal disease. JADA 1990; 121: $532-36$.

[8]. Klokkevold PR, Mealey BL, and Carranza FA. Influence of Systemic Disease and Disorders on the periodontium. In: Newman MG, Takei HH, Carranza FA, editors. Carranza's Clinical Periodontology. $9^{\text {th }}$ ed. Philadelphia, Pennsylvania: Saunders; 2003. pp 208-11.

[9]. Kiran M, Arpak N, Unsal E, Erdogan MF. The ffect of improved periodontal health on metabolic control in type 2 diabetes mellitus. J Clin Periodontol 2005;32 (3): $266-72$

[10]. Lamey PJ, Darwazeh AM, Frier BM.Oral disorders associated with diabetes mellitus. Diabet Med 1992; 9 (5): $410-16$.

[11]. Borghelli RF, Pettinari IL, Chuchurru JA, Stirparo MA. Oral lichen planus in patients with diabetes: An epidemiologic study. Oral Surg Oral Med Oral Pathol 1993; 75: 498 - 500. 
[12]. Vijayabala GS, Annigeri RG, Sudarshan R. Mucormycosis in a diabetic Ketoacidosis patient. Asian Pac J Trop Biomed 2013;3(10):830-33.

[13]. Farman AG. Atrophic lesions of the tongue: A prevalence study among 175 diabetic patients. J Oral Pathol 1976; 5: $255-64$.

[14]. Loe H. Periodontal disease. The sixth complication of diabetes mellitus. Diabetes Care 1993; $16: 329-34$. 